Homology, Homotopy and Applications, vol.7(2), 2005, pp.109-122

\title{
ON THE COBAR CONSTRUCTION OF A BIALGEBRA
}

\author{
T. KADEISHVILI
}

(communicated by Tom Lada)

\begin{abstract}
We show that the cobar construction of a DG-bialgebra is a homotopy G-algebra. This implies that the bar construction of this cobar is a DG-bialgebra as well.
\end{abstract}

\section{Introduction}

The cobar construction $\Omega C$ of a DG-coalgebra $(C, d: C \rightarrow C, \Delta: C \rightarrow C \otimes C)$ is, by definition, a DG-algebra. Suppose now that $C$ is additionally equipped with a multiplication $\mu: C \otimes C \rightarrow C$ turning $(C, d, \Delta, \mu)$ into a DG-bialgebra. How does this multiplication reflect on the cobar construction $\Omega C$ ? It was shown by Adams [1] that in the mod 2 situation in this case, the multiplication of $\Omega C$ is homotopy commutative: there exists a $\smile_{1}$ product

$$
\smile_{1}: \Omega C \otimes \Omega C \rightarrow \Omega C
$$

which satisfies the standard condition

$$
d\left(a \smile_{1} b\right)=d a \smile_{1} b+a \smile_{1} d b+a \cdot b+b \cdot a,
$$

(since we work mod 2 the signs are ignored in the whole paper). In this note we show that this $\smile_{1}$ gives rise to a sequence of operations

$$
E_{1, k}: \Omega C \otimes(\Omega C)^{\otimes k} \rightarrow \Omega C, k=1,2,3, \ldots
$$

which form on the cobar construction $\Omega C$ of a DG-bialgebra, a structure of homotopy $G$-algebra (hGa) in the sense of Gerstenhaber and Voronov [8].

There are two remarkable examples of homotopy G-algebras. The first one is the cochain complex of a 1-reduced simplicial set $C^{*}(X)$. The operations $E_{1, k}$ here are dual to cooperations defined by Baues in $[\mathbf{2}]$, and the starting operation $E_{1,1}$ is the classical Steenrod's $\smile_{1}$ product.

The second example is the Hochschild cochain complex $C^{*}(U, U)$ of an associative algebra $U$. The operations $E_{1, k}$ here were defined in [11] with the purpose of describing $A(\infty)$-algebras in terms of Hochschild cochains although the properties of those operations which were used as defining ones for the notion of homotopy Galgebra in [8] did not appear there. These operations were defined also in [9]. Again the starting operation $E_{1,1}$ is the classical Gerstenhaber's circle product which is sort of a $\smile_{1}$-product in the Hochschild complex.

Received November 13, 2003, revised December 1, 2003; published on April 22, 2005.

2000 Mathematics Subject Classification: 55P48.

Key words and phrases: Cobar construction, DG-bialgebra, Homotopy G-algebra.

(c) 2005, T. Kadeishvili. Permission to copy for private use granted. 
In this paper we present a third example of a homotopy G-algebra: we construct the operations $E_{1, k}$ on the cobar construction $\Omega C$ of a DG-bialgebra $C$, and the starting operation $E_{1,1}$ is again classical, it is Adams's $\smile_{1}$-product.

The notion of hGa was introduced in [8] as an additional structure on a DGalgebra $(A, d, \cdot)$ that induces a Gerstenhaber algebra structure on homology. The source of the defining identities and the main example was the Hochschild cochain complex $C^{*}(U, U)$. Another point of view is that hGa is a particular case of $B(\infty)$ algebra. This is an additional structure on a DG-algebra $(A, d, \cdot)$ that induces a DG-bialgebra structure on the bar construction $B A$.

We emphasize the third aspect of hGa: this is a structure which measures the noncommutativity of $A$. There exists the classical tool which measures the noncommutativity of a DG-algebra $(A, d, \cdot)$, namely the Steenrod's $\smile_{1}$ product, satisfying the condition (1). The existence of such $\smile_{1}$ guarantees the commutativity of $H(A)$, but the $\smile_{1}$ product satisfying just the condition (1) is too poor for most applications. In many constructions some deeper properties of $\smile_{1}$ are needed, for example the compatibility with the $\operatorname{dot}$ product of $A$ (the Hirsch formula)

$$
(a \cdot b) \smile_{1} c+a \cdot\left(b \smile_{1} c\right)+\left(a \smile_{1} c\right) \cdot b=0 .
$$

For a $\mathrm{hGa}\left(A, d, \cdot,\left\{E_{1, k}\right\}\right)$ the starting operation $E_{1,1}$ is a kind of $\smile_{1}$ product: it satisfies the conditions (1) and (2). As for the symmetric expression

$$
a \smile_{1}(b \cdot c)+b \cdot\left(a \smile_{1} c\right)+\left(a \smile_{1} b\right) \cdot c,
$$

it is just homotopical to zero and the appropriate homotopy is the operation $E_{1,2}$. The defining conditions of a hGa which satisfy higher operations $E_{1, k}$ can be regarded as generalized Hirsch formulas. So we can say that a hGa is a DG-algebra with a "good" $\smile_{1}$ product.

\section{Notation and preliminaries}

We work over $Z_{2}$. For a graded $Z_{2}$-module $M$ we denote by $s M$ the suspension of $M$, i.e. $(s M)^{i}=M^{i-1}$. Respectively $s^{-1} M$ denotes the desuspension of $M$, i.e. $\left(s^{-1} M\right)^{i}=M^{i+1}$. A differential graded algebra (DG-algebra) is a graded R-module $C=\left\{C^{i}\right\}, i \in Z$, with an associative multiplication $\mu: C^{i} \otimes C^{j} \rightarrow C^{i+j}$ and a homomorphism (a differential) $d: C^{i} \rightarrow C^{i+1}$ with $d^{2}=0$ and satisfying the Leibniz rule $d(x \cdot y)=d x \cdot y+x \cdot d y$, where $x \cdot y=\mu(x \otimes y)$. We assume that a DG-algebra contains a unit $1 \in C^{0}$. A non-negatively graded DG-algebra $C$ is connected if $C^{0}=Z_{2}$. A connected DG-algebra $C$ is $n$-reduced if $C^{i}=0,1 \leqslant i \leqslant n$. A DG-algebra is commutative if $\mu=\mu T$, where $T(x \otimes y)=y \otimes x$.

A differential graded coalgebra (DG-coalgebra) is a graded $Z_{2}$-module $C=$ $\left\{C_{i}\right\}, i \in Z$, with a coassociative comultiplication $\Delta: C \rightarrow C \otimes C$ and a homomorphism (a differential) $d: C_{i} \rightarrow C_{i+1}$ with $d^{2}=0$ and satisfying $\Delta d=$ $(d \otimes i d+i d \otimes d) \Delta$. A DG-coalgebra $C$ is assumed to have a counit $\epsilon: C \rightarrow$ $Z_{2}, \quad(\epsilon \otimes i d) \Delta=(i d \otimes \epsilon) \Delta=i d$. A non-negatively graded $\operatorname{dgc} C$ is connected if $C_{0}=Z_{2}$. A connected DG-coalgebra $C$ is n-reduced if $C_{i}=0,1 \leqslant i \leqslant n$. A differential graded bialgebra (DG-bialgebra) $(C, d, \mu, \Delta)$ is a DG-coalgebra $(C, d, \Delta)$ with a morphism of DG-coalgebras $\mu: C \otimes C \rightarrow C$ turning $(C, d, \mu)$ into a DG-algebra. 


\subsection{Cobar and Bar constructions}

Let $M$ be a graded $Z_{2}$-vector space with $M^{i \leqslant 0}=0$ and let $T(M)$ be the tensor algebra of $M$, i.e. $T(M)=\oplus_{i=0}^{\infty} M^{\otimes i}$.

$T(M)$ is a free graded algebra: for a graded algebra $A$ and a homomorphism $\alpha$ : $M \rightarrow A$ of degree zero there exists its multiplicative extension, a unique morphism of graded algebras $f_{\alpha}: T(M) \rightarrow A$ such that $f_{\alpha}(a)=\alpha(a)$. The map $f_{\alpha}$ is given by $f_{\alpha}\left(a_{1} \otimes \ldots \otimes a_{n}\right)=\alpha\left(a_{1}\right) \cdot \ldots \cdot \alpha\left(a_{n}\right)$. Dually, let $T^{c}(M)$ be the tensor coalgebra of $M$, i.e. $T^{c}(M)=\oplus_{i=0}^{\infty} M^{\otimes i}$, and the comultiplication $\nabla: T^{c}(M) \rightarrow T^{c}(M) \otimes T^{c}(M)$ is given by

$$
\nabla\left(a_{1} \otimes \ldots \otimes a_{n}\right)=\sum_{k=0}^{n}\left(a_{1} \otimes \ldots \otimes a_{k}\right) \otimes\left(a_{k+1} \otimes \ldots \otimes a_{n}\right) .
$$

$\left(T^{c}(M), \nabla\right)$ is a cofree graded coalgebra: for a graded coalgebra $C$ and a homomorphism $\beta: C \rightarrow M$ of degree zero there exists its comultiplicative extension, a unique morphism of graded coalgebras $g_{\beta}: C \rightarrow T^{c}(M)$ such that $p_{1} g_{\beta}=\beta$, here $p_{1}: T^{c}(M) \rightarrow M$ is the clear projection. The map $g_{\beta}$ is given by

$$
g_{\beta}(c)=\sum_{n} \beta\left(c^{(1)}\right) \otimes \ldots \otimes \beta\left(c^{(n)}\right),
$$

where $\Delta^{n}(c)=c^{(1)} \otimes \ldots \otimes c^{(n)}$ and $\Delta^{n}: C \rightarrow C^{\otimes n}$ is $n$-th iteration of the diagonal $\Delta: C \rightarrow C \otimes C$, i.e. $\Delta^{1}=i d, \Delta^{2}=\Delta, \Delta^{n}=\left(\Delta^{n-1} \otimes i d\right) \Delta$.

Let $\left(C, d_{C}, \Delta\right)$ be a connected DG-coalgebra and $\Delta=i d \otimes 1+1 \otimes i d+\Delta^{\prime}$. The (reduced) cobar construction $\Omega C$ on $C$ is a DG-algebra whose underlying graded algebra is $T\left(s C^{>0}\right)$. An element $\left(s c_{1} \otimes \ldots \otimes s c_{n}\right) \in(s C)^{\otimes n} \subset T\left(s C^{>0}\right)$ is denoted by $\left[c_{1}, \ldots, c_{n}\right] \in \Omega C$. The differential on $\Omega C$ is the sum $d=d_{1}+d_{2}$ which for a generator $[c] \in \Omega C$ is defined by $d_{1}[c]=\left[d_{C}(c)\right]$ and $d_{2}[c]=\sum\left[c^{\prime}, c^{\prime \prime}\right]$ for $\Delta^{\prime}(c)=\sum c^{\prime} \otimes c^{\prime \prime}$, and extended as a derivation. Let $\left(A, d_{A}, \mu\right)$ be a 1-reduced DG-algebra. The (reduced) bar construction $B A$ on $A$ is a DG-coalgebra whose underlying graded coalgebra is $T^{c}\left(s^{-1} A^{>0}\right)$. Again an element $\left(s^{-1} a_{1} \otimes \ldots \otimes s^{-1} a_{n}\right) \in\left(s^{-1} A\right)^{\otimes n} \subset T^{c}\left(s^{-1} A^{>0}\right)$ we denote as $\left[a_{1}, \ldots, a_{n}\right] \in B A$. The differential of $B A$ is the sum $d=d_{1}+d_{2}$ which for an element $\left[a_{1}, \ldots a_{n}\right] \in B A$ is defined by

$$
d_{1}\left[a_{1}, \ldots, a_{n}\right]=\sum_{i=1}^{n}\left[a_{1}, \ldots, d_{A} a_{i}, \ldots, a_{n}\right], d_{2}\left[a_{1}, \ldots, a_{n}\right]=\sum_{i=1}^{n-1}\left[a_{1}, \ldots, a_{i} \cdot a_{i+1}, \ldots, a_{n}\right] .
$$

\subsection{Twisting cochains}

Let $(C, d, \Delta)$ be a dgc, $(A, d, \mu)$ a dga. A twisting cochain [5] is a homomorphism $\tau: C \rightarrow A$ of degree +1 satisfying the Browns' condition

$$
d \tau+\tau d=\tau \smile \tau,
$$

where $\tau \smile \tau^{\prime}=\mu_{A}\left(\tau \otimes \tau^{\prime}\right) \Delta$. We denote by $T(C, A)$ the set of all twisting cochains $\tau: C \rightarrow A$.

There are universal twisting cochains $C \rightarrow \Omega C$ and $B A \rightarrow A$ being clear inclusion and projection respectively. Here are essential consequences of the condition (3):

(i) The multiplicative extension $f_{\tau}: \Omega C \rightarrow A$ is a map of DG-algebras, so there is a bijection $T(C, A) \leftrightarrow H^{\circ o m} m_{D-A l g}(\Omega C, A)$; 
(ii) The comultiplicative extension $g_{\tau}: C \rightarrow B A$ is a map of DG-coalgebras, so there is a bijection $T(C, A) \leftrightarrow \operatorname{Hom}_{D G-\text { Coalg }}(C, B A)$.

\section{Homotopy G-algebras}

\subsection{Products in the bar construction}

Let $(A, d, \cdot)$ be a 1-reduced DG-algebra and $B A$ its bar construction. We are interested in the structure of a multiplication

$$
\mu: B A \otimes B A \rightarrow B A,
$$

turning $B A$ into a DG-bialgebra, i.e. we require that

(i) $\mu$ is a DG-coalgebra map;

(ii) is associative;

(iii) has the unit element $1_{\Lambda} \in \Lambda \subset B A$.

Because of the cofreeness of the tensor coalgebra $B A=T^{c}\left(s^{-1} A\right)$, a map of graded coalgebras

$$
\mu: B A \otimes B A \rightarrow B A
$$

is uniquely determined by the projection of degree +1

$$
E=p r \cdot \mu: B A \otimes B A \rightarrow B A \rightarrow A .
$$

Conversly, a homomorphism $E: B A \otimes B A \rightarrow A$ of degree +1 determines its coextension, a graded coalgebra map $\mu_{E}: B A \otimes B A \rightarrow B A$ given by

$$
\mu_{E}=\sum_{k=0}^{\infty}(E \otimes \ldots \otimes E) \nabla_{B A \otimes B A}^{k},
$$

where $\nabla_{B A \otimes B A}^{k}: B A \otimes B A \rightarrow(B A \otimes B A)^{\otimes k}$ is the k-fold iteration of the standard coproduct of tensor product of coalgebras

$$
\nabla_{B A \otimes B A}=(i d \otimes T \otimes i d)(\nabla \otimes \nabla): B A \otimes B A \rightarrow(B A \otimes B A)^{\otimes 2} .
$$

The map $\mu_{E}$ is a chain map (i.e. it is a map of DG-coalgebras) if and only if $E$ is a twisting cochain in the sense of E. Brown, i.e. satisfies the condition

$$
d E+E d_{B A \otimes B A}=E \smile E .
$$

Indeed, again because of the cofreeness of the tensor coalgebra $B A=T^{c}\left(s^{-1} A\right)$ the condition $d_{B A} \mu_{E}=\mu_{E} d_{B A \otimes B A}$ is satisfied if and only if it is satisfied after the projection on $A$, i.e. if $p r \cdot d_{B A} \mu_{E}=p r \cdot \mu_{E} d_{B A \otimes B A}$ but this condition is nothing else than the Brown's condition (4).

The same argument shows that the product $\mu_{E}$ is associative if and only if $p r \cdot \mu_{E}\left(\mu_{E} \otimes i d\right)=p r \cdot \mu_{E}\left(i d \otimes \mu_{E}\right)$, or, having in mind $E=p r \cdot \mu_{E}$

$$
E\left(\mu_{E} \otimes i d\right)=E\left(i d \otimes \mu_{E}\right) .
$$

A homomorphism $E: B A \otimes B A \rightarrow A$ consists of components

$$
\left\{\bar{E}_{p, q}:\left(s^{-1} A\right)^{\otimes p} \otimes\left(s^{-1} A\right)^{\otimes q} \rightarrow A, p, q=0,1,2, \ldots\right\},
$$


where $\bar{E}_{p q}$ is the restriction of $E$ on $\left(s^{-1} A\right)^{\otimes p} \otimes\left(s^{-1} A\right)^{\otimes q}$. Each component $\bar{E}_{p, q}$ can be regarded as an operation

$$
E_{p, q}: A^{\otimes p} \otimes A^{\otimes q} \rightarrow A, p, q=0,1,2, \ldots .
$$

The value of $E_{p, q}$ on the element $\left(a_{1} \otimes \ldots \otimes a_{p}\right) \otimes\left(b_{1} \otimes \ldots \otimes b_{q}\right)$ we denote by $E_{p, q}\left(a_{1}, \ldots, a_{p} ; b_{1} \ldots, b_{q}\right)$.

It is not hard to check that the multiplication $\mu_{E}$ induced by $E$ (or equivalently by a collection of multioperations $\left\{E_{p, q}\right\}$ ) has the unit $1_{\Lambda} \in \Lambda \subset B A$ if and only if

$$
E_{0,1}=E_{1,0}=i d ; \quad E_{0, k}=E_{k, 0}=0, k>1 .
$$

So we can summarize:

Proposition 1. The multiplication $\mu_{E}$ induced by a collection of multioperations $\left\{E_{p, q}\right\}$ turns $B A$ into a DG-bialgebra, i.e. satisfies (i-iii), if and only if the conditions (4), (5), and (6) are satisfied.

Let us interpret the condition (4) in terms of the components $E_{p q}$. The restriction of (4) on $A \otimes A$ gives

$$
d E_{1,1}(a ; b)+E_{1,1}(d a ; b)+E_{1,1}(a ; d b)=a \cdot b+b \cdot a .
$$

This condition coincides with the condition (1), i.e. the operation $E_{1,1}$ is sort of a $\smile_{1}$ product, which measures the noncommutativity of $A$. Below we denote $E_{1,1}(a ; b)=$ $a \smile_{1} b$.

The restriction on $A^{\otimes 2} \otimes A$ gives

$$
\begin{gathered}
d E_{2,1}(a, b ; c)+E_{2,1}(d a, b ; c)+E_{2,1}(a, d b ; c)+E_{2,1}(a, b ; d c)= \\
(a \cdot b) \smile_{1} c+a \cdot\left(b \smile_{1} c\right)+\left(a \smile_{1} c\right) \cdot b,
\end{gathered}
$$

this means, that this $\smile_{1}$ satisfies the left Hirsch formula (2) up to homotopy and the appropriate homotopy is the operation $E_{2,1}$.

The restriction on $A \otimes A^{\otimes 2}$ gives:

$$
\begin{gathered}
d E_{1,2}(a ; b, c)+E_{1,2}(d a ; b, c)+E_{1,2}(a ; d b, c)+E_{1,2}(a ; b, d c)= \\
a \smile_{1}(b \cdot c)+\left(a \smile_{1} b\right) \cdot c+b \cdot\left(a \smile_{1} c\right),
\end{gathered}
$$

this means, that this $\smile_{1}$ satisfies the right Hirsch formula (2) up to homotopy and the appropriate homotopy is the operation $E_{1,2}$.

Generally the restriction of (4) on $A^{\otimes m} \otimes A^{\otimes n}$ gives:

$$
\begin{gathered}
d E_{m, n}\left(a_{1}, \ldots, a_{m} ; b_{1}, \ldots, b_{n}\right)+\sum_{i} E_{m, n}\left(a_{1}, \ldots, d a_{i}, \ldots, a_{m} ; b_{1}, \ldots, b_{n}\right) \\
\quad+\sum_{i} E_{m, n}\left(a_{1}, \ldots, a_{m} ; b_{1}, \ldots, d b_{i}, \ldots, b_{n}\right)= \\
a_{1} \cdot E_{m-1, n}\left(a_{2}, \ldots, a_{m} ; b_{1}, \ldots, b_{n}\right)+E_{m-1, n}\left(a_{1}, \ldots, a_{m-1} ; b_{1}, \ldots, b_{n}\right) \cdot a_{m} \\
+b_{1} \cdot E_{m, n-1}\left(a_{1}, \ldots, a_{m} ; b_{2}, \ldots, b_{n}\right)+E_{m, n-1}\left(a_{1}, \ldots, a_{m} ; b_{1}, \ldots, b_{n-1}\right) \cdot b_{m}+ \\
\sum_{i} E_{m-1, n}\left(a_{1}, \ldots, a_{i} \cdot a_{i+1}, \ldots, a_{m} ; b_{1}, \ldots, b_{n}\right)+ \\
\sum_{i} E_{m, n-1}\left(a_{1}, \ldots, a_{m} ; b_{1}, \ldots, b_{i} \cdot b_{i+1}, \ldots, b_{n}\right)+ \\
\sum_{p=1}^{m-1} \sum_{q=1}^{n-1} E_{p, q}\left(a_{1}, \ldots, a_{p} ; b_{1}, \ldots, b_{q}\right) \cdot E_{m-p, n-q}\left(a_{p+1}, \ldots, a_{m} ; b_{q+1}, \ldots, b_{n}\right) .
\end{gathered}
$$

Now let us interpret the associativity condition (5) in terms of the components 
$E_{p, q}$. The restriction of (5) on $A \otimes A \otimes A$ gives

$$
\begin{gathered}
\left(a \smile_{1} b\right) \smile_{1} c+a \smile_{1}\left(b \smile_{1} c\right)=E_{1,2}(a ; b, c)+E_{1,2}(a ; c, b)+ \\
E_{2,1}(a, b ; c)+E_{2,1}(b, a ; c) .
\end{gathered}
$$

Generally the restriction of (5) on $A^{\otimes k} \otimes A^{\otimes l} \otimes A^{\otimes m}$ gives

$$
\begin{gathered}
\sum_{r=1}^{l+m} \sum_{l_{1}+\ldots+l_{r}=l, m_{1}+\ldots+m_{r}=m} \\
E_{k, r}\left(a_{1}, \ldots, a_{k} ; E_{l_{1}, m_{1}}\left(b_{1}, \ldots, b_{l_{1}} ; c_{1}, \ldots, c_{m_{1}}\right), \ldots,\right. \\
E_{l_{r}, m_{r}}\left(b_{l_{1}+\ldots+l_{r}+1+1}, \ldots, b_{l} ; c_{m_{1}+\ldots+m_{r-1}+1}, \ldots, c_{m}\right)= \\
\sum_{s+1}^{k+l} \sum_{k_{1}+\ldots+k_{s}=k, l_{1}+\ldots+l_{s}=l} \\
E_{s, m}\left(E_{k_{1} l_{1}}\left(a_{1}, \ldots, a_{k_{1}} ; b_{1}, \ldots, b_{l_{1}}\right), \ldots,\right. \\
\left.E_{k_{s}, l_{s}}\left(a_{k_{1}+\ldots+k_{s-1}+1}, \ldots, a_{k} ; b_{l_{1}+\ldots+l_{s-1}+1}, \ldots, b_{l}\right) ; c_{1}, \ldots, c_{m}\right)
\end{gathered}
$$

We define a Hirsch algebra as a DG-algebra $(A, d, \cdot)$ endowed with a sequence of multioperations $\left\{E_{p, q}\right\}$ satisfying (6), (10). This name is inspired by the fact that the defining condition (10) can be regarded as generalizations of classical Hirsch formula (2). This notion was used in $[\mathbf{1 2}],[\mathbf{1 3}]$.

A Hirsch algebra we call associative if in addition the condition (12) is satisfied.

This structure is a particular case of a $B_{\infty}$-algebra, see below. Moreover, the notion of homotopy $G$-algebra, described below, is a particular case of an associative Hirsch algebra.

\subsection{Some particular cases}

For a Hirsch algebra $\left(A, d, \cdot,\left\{E_{p, q}\right\}\right)$ the operation $E_{1,1}=\smile_{1}$ satisfies (1), so this structure can be considered as a tool which measures the noncommutativity of the product $a \cdot b$ of $A$. We distinguish various levels of "noncommutativity" of $A$ according to the form of $\left\{E_{p, q}\right\}$.

Level 1. Suppose for the collection $\left\{E_{p, q}\right\}$ all the operations except $E_{0,1}=i d$ and $E_{1,0}=i d$ are trivial. Then it follows from (7) that in this case $A$ is a strictly commutative DG-algebra.

Level 2. Suppose all operations except $E_{0,1}=i d, E_{1,0}=i d$ and $E_{1,1}$ are trivial. In this case $A$ is endowed with a "strict" $\smile_{1}$ product $a \smile_{1} b=E_{1,1}(a ; b)$ : the condition (10) here degenerate to the following 4 conditions

$$
\begin{gathered}
d\left(a \smile_{1} b\right)=d a \smile_{1} b+a \smile_{1} d b+a \cdot b+b \cdot a, \\
(a \cdot b) \smile_{1} c+a \cdot\left(b \smile_{1} c\right)+\left(a \smile_{1} c\right) \cdot b=0, \\
a \smile_{1}(b \cdot c)+b \cdot\left(a \smile_{1} c\right)+\left(a \smile_{1} b\right) \cdot c=0, \\
\left(a \smile_{1} c\right) \cdot\left(b \smile_{1} d\right)=0 .
\end{gathered}
$$

The condition (12) degenerates to the associativity $\smile_{1}$

$$
a \smile_{1}\left(b \smile_{1} c\right)=\left(a \smile_{1} b\right) \smile_{1} c .
$$

As we see in this case we have very strong restrictions on the $\smile_{1}$-product. An example of a DG-algebra with such strict $\smile_{1}$ product is $\left(H^{*}\left(S X, Z_{2}\right), d=0\right)$ with 
$a \smile_{1} b=0$ if $a \neq b$ and $a \smile_{1} a=S q^{|a|-1} a$; another example is $C^{*}(S X, C X)$, where $S X$ is the suspension and $C X$ is the cone of a space $X$ (see [18]).

Level 3. Suppose all operations except $E_{0,1}=i d, E_{1,0}=i d$ and $E_{1, k}, k=1,2,3, \ldots$ are trivial. In this case the condition (10) degenerates into two conditions: at $A \otimes A^{\otimes k}$

$$
\begin{gathered}
d E_{1, k}\left(a ; b_{1}, \ldots, b_{k}\right)+E_{1, k}\left(d a ; b_{1}, \ldots, b_{k}\right)+\sum_{i} E_{1, k}\left(a ; b_{1}, \ldots, d b_{i}, \ldots, b_{k}\right)= \\
b_{1} \cdot E_{1, k-1}\left(a ; b_{2}, \ldots, b_{k}\right)+E_{1, k-1}\left(a ; b_{1}, \ldots, b_{k-1}\right) \cdot b_{k}+ \\
\sum_{i} E_{1, k-1}\left(a ; b_{1}, \ldots, b_{i} \cdot b_{i+1}, \ldots, b_{k}\right)
\end{gathered}
$$

and at $A^{\otimes 2} \otimes A^{\otimes k}$

$$
\begin{gathered}
E_{1, k}\left(a_{1} \cdot a_{2} ; b_{1}, \ldots, b_{k}\right)=a_{1} \cdot E_{1, k}\left(a_{2} ; b_{1}, \ldots, b_{k}\right)+E_{1, k}\left(a_{1} ; b_{1}, \ldots, b_{k}\right) \cdot a_{2}+ \\
\sum_{p=1}^{k-1} E_{1, p}\left(a_{1} ; b_{1}, \ldots, b_{p}\right) \cdot E_{1, m-p}\left(a_{2} ; b_{p+1}, \ldots, b_{k}\right) ;
\end{gathered}
$$

moreover at $A^{\otimes n>2} \otimes A^{\otimes k}$ the condition is trivial. In particular the condition (8) here degenerates to Hirsch formula (2).

The associativity condition (12) in this case looks like

$$
\begin{gathered}
E_{1, n}\left(E_{1, m}\left(a ; b_{1}, \ldots, b_{m}\right) ; c_{1}, \ldots, c_{n}\right)=\sum_{0 \leqslant i_{1} \leqslant \ldots \leqslant i_{m} \leqslant n} \sum_{0 \leqslant n_{1}+\ldots+n_{r} \leqslant n} \\
E_{1, n-\left(n_{1}+\ldots+n_{j}\right)+j}\left(a ; c_{1}, \ldots, c_{i_{1}}, E_{1, n_{1}}\left(b_{1} ; c_{i_{1}+1}, \ldots, c_{i_{1}+n_{1}}\right), c_{i_{1}+n_{1}+1}, \ldots,\right. \\
c_{i_{2}}, E_{1, n_{2}}\left(b_{2} ; c_{i_{2}+1}, \ldots, c_{i_{2}+n_{2}}\right), c_{i_{2}+n_{2}+1}, \ldots, \\
\left.c_{i_{m}}, E_{1, n_{m}}\left(b_{m} ; c_{i_{m}+1}, \ldots, c_{i_{m}+n_{m}}\right), c_{i_{m}+n_{m}+1}, \ldots, c_{n}\right),
\end{gathered}
$$

In particular the condition (11) here degenerates to

$$
\left(a \smile_{1} b\right) \smile_{1} c+a \smile_{1}\left(b \smile_{1} c\right)=E_{1,2}(a ; b, c)+E_{1,2}(a ; c, b) .
$$

The structure of this level coincides with the notion of Homotopy G-algebra, see below.

Level 4. As the last level we consider a Hirsch algebra structure with no restrictions. An example of such structure is the cochain complex of a 1-reduced cubical set. Note that it is a nonassociative Hirsch algebra.

\section{3. $\quad B_{\infty}$-algebra}

The notion of a $B_{\infty}$-algebra was introduced in [2], [10] as an additional structure on a DG-algebra $(A, \cdot, d)$ which turns the tensor coalgebra $T^{c}\left(s^{-1} A\right)=B A$ into a DG-bialgebra. So it requires a new differential

$$
\widetilde{d}: B A \rightarrow B A
$$

(which should be a coderivation with respect to standard coproduct of $B A$ ) and a new associative multiplication

$$
\widetilde{\mu}:(B A, \widetilde{d}) \otimes(B A, \widetilde{d}) \rightarrow(B A, \widetilde{d})
$$

which should be a map of DG-coalgebras, with $1_{\Lambda} \in \Lambda \subset B A$ as the unit element.

It is known that such $\widetilde{d}$ specifies on $A$ a structure of $A_{\infty}$-algebra in the sense of Stasheff [19], namely a sequence of operations $\left\{m_{i}: \otimes^{i} A \rightarrow A, i=1,2,3, \ldots\right\}$ subject of appropriate conditions.

As for the new multiplication $\widetilde{\mu}$, it follows from the above considerations, that it is induced by a sequence of operations $\left\{E_{p q}\right\}$ satisfying (6), (12) and the modified condition (10) with involved $A_{\infty}$-algebra structure $\left\{m_{i}\right\}$. 
Thus the structure of associative Hirsch algebra is a particular $B_{\infty}$-algebra structure on $A$ when the standard differential of the bar construction $d_{B}: B A \rightarrow B A$ does not change, i.e. $\widetilde{d}=d_{B}$ (in this case the corresponding $A_{\infty}$-algebra structure is degenerate: $\left.m_{1}=d_{A}, m_{2}=\cdot m_{3}=0, m_{4}=0, \ldots\right)$.

Let us mention that a twisting cochain $E$ satisfying (6) and (4), (but not (5) i.e. the induced product in the bar construction is not strictly associative), was constructed in [14] for the singular cochain complex of a topological space $C^{*}(X)$ using acyclic models. The condition (6) determines this twisting cochain $E$ uniquely up to standard equivalence (homotopy) of twisting cochains in the sense of $\mathrm{N}$. Berikashvili [4].

\subsection{Strong homotopy commutative algebras}

The notion of strong homotopy commutative algebra (shc-algebra), as a tool for measuring of noncommutativity of DG-algebras, was used in many papers: [17], [20], etc.

A shc-algebra is a DG-algebra $(A, d, \cdot)$ with a given twisting cochain $\Phi: B(A \otimes$ $A) \rightarrow A$ which satisfies apropriate up to homotopy conditions of associativity and commutativity. Compare with the Hirsch algebra structure which is represented by a twisting cochain $E: B A \otimes B A \rightarrow A$. Standard contraction of $B(A \otimes A)$ to $B A \otimes B A$ allows one to establish a connection between these two notions.

\subsection{DG-Lie algebra structure in a Hirsch algebra}

A structure of an associative Hirsch algebra on $A$ induces on the homology $H(A)$ a structure of Gerstenhaber algebra (G-algebra) (see [6], [8], [21]) which is defined as a commutative graded algebra $(H, \cdot)$ together with a Lie bracket of degree -1

$$
[,]: H^{p} \otimes H^{q} \rightarrow H^{p+q-1}
$$

(i.e. a graded Lie algebra structure on the desuspension $s^{-1} H$ ) that is a biderivation: $[a, b \cdot c]=[a, b] \cdot c+b \cdot[a, c]$.

The existence of this structure in the homology $H(A)$ is seen by the following argument.

Let $\left(A, d, \cdot,\left\{E_{p, q}\right\}\right)$ be an associative Hirsch algebra, then in the desuspension $s^{-1} A$ there appears a structure of DG-Lie algebra: although the $\smile_{1}=E_{1,1}$ is not associative, the condition (11) implies the pre-Jacobi identity

$$
a \smile_{1}\left(b \smile_{1} c\right)+\left(a \smile_{1} b\right) \smile_{1} c=a \smile_{1}\left(c \smile_{1} b\right)+\left(a \smile_{1} c\right) \smile_{1} b
$$

This condition guarantees that the commutator $[a, b]=a \smile_{1} b+b \smile_{1} a$ satisfies the Jacobi identity. Besides, condition (7) implies that [, ] : $A^{p} \otimes A^{q} \rightarrow A^{p+q-1}$ is a chain map. Thus on $s^{-1} H(A)$ there appears the structure of graded Lie algebra. The up to homotopy Hirsh formulae (8) and (9) imply that the induced Lie bracket is a biderivation.

\subsection{Homotopy G-algebra}

An associative Hirsch algebra of level 3 in the literature is known as Homotopy G-algebra . 
A Homotopy G-algebra in [8] and [21] is defined as a DG-algebra $(A, d, \cdot)$ with a given sequence of multibraces $a\left\{a_{1}, \ldots, a_{k}\right\}$ which, in our notation, we regard as a sequence of operations

$$
E_{1, k}: A \otimes\left(\otimes^{k} A\right) \rightarrow A, \quad k=0,1,2,3, \ldots
$$

which, together with $E_{01}=i d$ satisfies the conditions (6), (13), (14) and (15).

The name Homotopy G-algebra is motivated by the fact that this structure induces on the homology $H(A)$ the structure of G-algebra (as we have seen in the previous section such a structure appears even on the homology of an associative Hirsch algebra).

The conditions (13), (14), and (15) in [8] are called higher homotopies, distributivity and higher pre-Jacobi identities respectively. As we have seen the first two conditions mean that $E: B A \otimes B A \rightarrow A$ is a twisting cochain, or equivalently $\mu_{E}: B A \otimes B A \rightarrow B A$ is a chain map, and the third one means that this multiplication is associative.

\subsection{Operadic description}

Appropriate language to describe such huge sets of operations is the operadic language. Here we use the surjection operad $\chi$ and the Barratt-Eccles operad $\mathcal{E}$ which are the most convenient $E_{\infty}$ operads. For definitions we refer to [3].

The operations $E_{1, k}$ forming hGa have nice description in the surjection operad, see $[\mathbf{1 5}],[\mathbf{1 6}],[\mathbf{3}]$. Namely, to the dot product corresponds the element $(1,2) \in \chi_{0}(2)$, to $E_{1,1}=\smile_{1}$ product corresponds $(1,2,1) \in \chi_{1}(2)$, to the operation $E_{1,2}$ the element $(1,2,1,3) \in \chi_{2}(3)$, etc. Generally to the operation $E_{1, k}$ corresponds the element

$$
E_{1, k}=(1,2,1,3, \ldots, 1, k, 1, k+1,1) \in \chi_{k}(k+1) .
$$

We remark here that the defining conditions of a hGa (13), (14), (15) can be expressed in terms of operadic structure (differential, symmetric group action and composition product) and the elements (17) satisfy these conditions already in the operad $\chi$. This in particular implies that any $\chi$-algebra is automatically a $h G a$. Note that the elements $(17)$ together with $(1,2)$ generate the suboperad $F_{2} \chi$ which is equivalent to the little square operad. This fact and a hGa structure on the Hochschild cochain complex $C^{*}(U, U)$ of an algebra $U$ are used by many authors to prove so called Deligne conjecture about the action of the little square operad on $C^{*}(U, U)$.

Now look at the operations $E_{p, q}$ which define a structure of Hirsch algebra. They can not live in $\chi$ : it is enough to mention that the Hirsch formula (2), as a part of defining conditions of $\mathrm{hGa}$, is satisfied in $\chi$, but for a Hirsch algebra this condition is satisfied up to homotopy $E_{2,1}$, see (8). We belive that $E_{p, q^{-}}$s live in the BarrattEccles operad $\mathcal{E}$. In particular direct calculation shows that

$$
\begin{aligned}
& E_{1,1}=((1,2),(2,1)) \in \mathcal{E}_{1}(2) ; \\
& E_{1,2}=((\mathbf{1}, 2,3),(2, \mathbf{1}, 3),(2,3, \mathbf{1})) \in \mathcal{E}_{2}(3) ; \\
& E_{2,1}=((1,2, \mathbf{3}),(1, \mathbf{3}, 2),(\mathbf{3}, 1,2)) \in \mathcal{E}_{2}(3) ; \\
& E_{1,3}=((\mathbf{1}, 2,3,4),(2, \mathbf{1}, 3,4),(2,3, \mathbf{1}, 4),(2,3,4, \mathbf{1})) \in \mathcal{E}_{3}(4) ; \\
& E_{3,1}=((1,2,3, \mathbf{4}),(1,2, \mathbf{4}, 3),(1, \mathbf{4}, 2,3),(\mathbf{4}, 1,2,3)) \in \mathcal{E}_{3}(4)
\end{aligned}
$$


and in general

$$
\begin{aligned}
& E_{1, k}=((\mathbf{1}, 2, \ldots, k+1), \ldots,(2,3, \ldots, i, \mathbf{1}, i+1, \ldots, k+1), \ldots,(2,3, \ldots, k+1, \mathbf{1})) \\
& E_{k, 1}=((1,2, \ldots, \mathbf{k}+\mathbf{1}), \ldots,(1,2, \ldots, i, \mathbf{k}+\mathbf{1}, i+1, \ldots, k), \ldots,(\mathbf{k}+\mathbf{1}, 1,2, \ldots, k)) .
\end{aligned}
$$

As for other $E_{p, q}$-s we can indicate just

$$
\begin{aligned}
E_{2,2}= & ((1,2,3,4),(1,3,4,2),(3,1,4,2),(3,4,1,2))+ \\
& ((1,2,3,4),(3,1,2,4),(3,1,4,2),(3,4,1,2))+ \\
& ((1,2,3,4),(1,3,2,4),(1,3,4,2),(3,1,4,2))+ \\
& ((1,2,3,4),(1,3,2,4),(3,1,2,4),(3,1,4,2)) .
\end{aligned}
$$

We remark that the operadic table reduction map $T R: \mathcal{E} \rightarrow \chi$, see [3], maps $E_{k>1,1}$ and $E_{2,2}$ to zero, and $E_{1, k} \in \mathcal{E}_{k}(k+1)$ to $E_{1, k} \in \chi_{k}(k+1)$.

\section{Adams $\smile_{1}$-product in the cobar construction of a bialge- bra}

Here we present the Adams $\smile_{1}$-product $\smile_{1}: \Omega A \otimes \Omega A \rightarrow \Omega A$ on the cobar construction $\Omega A$ of a DG-bialgebra $(A, d, \Delta: A \rightarrow A \otimes A, \mu: A \otimes A \rightarrow A)$ (see [1]). This will be the first step in the construction of an hGa structure on $\Omega A$.

This $\smile_{1}$ product satisfies the Steenrod condition (1) and the Hirsch formula (2).

First we define the $\smile_{1}$-product of two elements $x=[a], y=[b] \in \Omega A$ of length 1 as $[a] \smile_{1}[b]=[a \cdot b]$. Extending this definition by (2) we obtain

$$
\begin{gathered}
{\left[a_{1}, a_{2}\right] \smile_{1}[b]=\left(\left[a_{1}\right] \cdot\left[a_{2}\right]\right) \smile_{1}[b]=\left[a_{1}\right] \cdot\left(\left[a_{2}\right] \smile_{1}[b]\right)+\left(\left[a_{1}\right] \smile_{1}[b]\right) \cdot\left[a_{2}\right]=} \\
{\left[a_{1}\right] \cdot\left[a_{2} \cdot b\right]+\left[a_{1} \cdot b\right] \cdot\left[a_{2}\right]=\left[a_{1}, a_{2} \cdot b\right]+\left[a_{1} \cdot b, a_{2}\right] .}
\end{gathered}
$$

Further iteration of this process gives

$$
\left[a_{1}, \ldots, a_{n}\right] \smile_{1}[b]=\sum_{i}\left[a_{1}, \ldots, a_{i-1}, a_{i} \cdot b, a_{i+1}, \ldots, a_{n}\right] .
$$

Now let's define $[a] \smile_{1}\left[b_{1}, b_{2}\right]=\left[a^{(1)} \cdot b, a^{(2)} \cdot b\right]$ where $\Delta a=a^{(1)} \otimes a^{(2)}$ is the value of the diagonal $\Delta: A \rightarrow A \otimes A$ on $[a]$. Inspection shows that the condition (1) for short elements

$$
d\left([a] \smile_{1}[b]\right)=d[a] \smile_{1}[b]+[a] \smile_{1} d[b]+[a] \cdot[b]+[b] \cdot[a] .
$$

is satisfied.

Generally we define the $\smile_{1}$ product of an element $x=[a] \in \Omega A$ of length 1 and an element $y=\left[b_{1}, \ldots, b_{n}\right] \in \Omega A$ of arbitrary length by

$$
[a] \smile_{1}\left[b_{1}, \ldots, b_{n}\right]=\left[a^{(1)} \cdot b_{1}, \ldots, a^{(n)} \cdot b_{n}\right] ;
$$

here $\Delta^{n}(a)=a^{(1)} \otimes \ldots \otimes a^{(n)}$ is the $\mathrm{n}$-fold iteration of the diagonal $\Delta: A \rightarrow A \otimes A$ and $a \cdot b=\mu(a \otimes b)$ is the product in $A$.

Extending this definition for the elements of arbitrary lengths $\left[a_{1}, \ldots, a_{m}\right] \smile_{1}$ $\left[b_{1}, \ldots, b_{n}\right]$ by the Hirsch formula (2) we obtain the general formula

$$
\left[a_{1}, \ldots, a_{m}\right] \smile_{1}\left[b_{1}, \ldots, b_{n}\right]=\sum_{k}\left[a_{1}, \ldots, a_{k-1}, a_{k}^{(1)} \cdot b_{1}, \ldots, a_{k}^{(n)} \cdot b_{n}, a_{k+1}, \ldots, a_{m}\right] .
$$


Of course, so defined, the $\smile_{1}$ satisfies the Hirsch formula (2) automatically. It remains to prove the

Proposition 2. This $\smile_{1}$ satisfies Steenrod condition (1)

$$
\begin{gathered}
d_{\Omega}\left(\left[a_{1}, \ldots, a_{m}\right] \smile_{1}\left[b_{1}, \ldots, b_{n}\right]\right)= \\
d_{\Omega}\left[a_{1}, \ldots, a_{m}\right] \smile_{1}\left[b_{1}, \ldots, b_{n}\right]+\left[a_{1}, \ldots, a_{m}\right] \smile_{1} d_{\Omega}\left[b_{1}, \ldots, b_{n}\right]+ \\
{\left[a_{1}, \ldots, a_{m}, b_{1}, \ldots, b_{n}\right]+\left[b_{1}, \ldots, b_{n}, a_{1}, \ldots, a_{m}\right] .}
\end{gathered}
$$

Proof. Let us denote this condition by Steen $_{m, n}$. The first step consists in direct checking of the conditions Steen $_{1, m}$ by induction on $m$. Furthermore, assume that Steen $_{m, n}$ is satisfied. Let us check the condition Steen $_{m+1, n}$ for $\left[a, a_{1}, \ldots, a_{m}\right] \smile_{1}$ $\left[b_{1}, \ldots, b_{n}\right]$. We denote $\left[a_{1}, \ldots, a_{m}\right]=x,\left[b_{1}, \ldots, b_{m}\right]=y$. Using the Hirsch formula (2), Steen $_{m, n}$, and Steen $_{1, n}$ we obtain:

$$
\begin{gathered}
d\left(\left[a, a_{1}, \ldots, a_{m}\right] \smile_{1}\left[b_{1}, \ldots, b_{n}\right]\right)= \\
d\left(([a] \cdot x) \smile_{1} y\right)=d\left([a] \cdot\left(x \smile_{1} y\right)+\left([a] \smile_{1} y\right) \cdot x\right)= \\
=d[a] \cdot\left(x \smile_{1} y\right)+[a] \cdot\left(d x \smile_{1} y+x \smile_{1} d y+x \cdot y+y \cdot x\right)+ \\
\left(d[a] \smile_{1} y+[a] \smile_{1} d y+[a] \cdot y+y \cdot[a]\right) \cdot x+\left([a] \smile_{1} y\right) d x= \\
d[a] \cdot\left(x \smile_{1} y\right)+[a] \cdot\left(d x \smile_{1} y\right)+[a] \cdot\left(x \smile_{1} d y\right)+[a] \cdot x \cdot y+[a] \cdot y \cdot x+ \\
\left(d[a] \smile_{1} y \cdot\right) x+\left([a] \smile_{1} d y \cdot\right) x+[a] \cdot y \cdot x+y \cdot[a] \cdot x+\left([a] \smile_{1} y\right) d x .
\end{gathered}
$$

Besides, using Hirsch (2) formula we obtain

$$
\begin{gathered}
d\left[a, a_{1}, \ldots, a_{m}\right] \smile_{1}\left[b_{1}, \ldots, b_{n}\right]= \\
d([a] \cdot x) \smile_{1} y=(d[a] \cdot x) \smile_{1} y+([a] \cdot d x) \smile_{1} y= \\
d[a] \cdot\left(x \smile_{1} y\right)+\left(d[a] \smile_{1} y\right) \cdot x+[a] \cdot\left(d x \smile_{1} y\right)+\left([a] \smile_{1} y\right) \cdot d x
\end{gathered}
$$

and

$$
\begin{gathered}
{\left[a, a_{1}, \ldots, a_{m}\right] \smile_{1} d\left[b_{1}, \ldots, b_{n}\right]=} \\
([a] \cdot x) \smile_{1} d y=[a] \cdot\left(x \smile_{1} d y\right)+\left([a] \smile_{1} d y\right) \cdot x,
\end{gathered}
$$

now it is evident that Steen $_{m+1, n}$ is satisfied. This completes the proof.

\section{Homotopy G-algebra structure on the cobar construction of a bialgebra}

Below we present a sequence of operations

$$
E_{1, k}: \Omega A \otimes(\Omega A)^{\otimes k} \rightarrow \Omega A,
$$

which extends the above described $E_{1,1}=\smile_{1}$ to a structure of a homotopy G-algebra on the cobar construction of a DG-bialgebra. This means that $E_{1, k}$-s satisfy the conditions (13), (14) and (15). 
For $x=[a] \in \Omega A$ of length $1, y_{i} \in \Omega A$ and $k>1$ we define $E_{1, k}\left([a] ; y_{1}, \ldots y_{k}\right)=0$ and extend for an arbitrary $x=\left[a_{1}, \ldots a_{n}\right]$ by (14). This gives

$$
E_{1, k}\left(\left[a_{1}, \ldots, a_{n}\right] ; y_{1}, \ldots, y_{k}\right)=0
$$

for $n<k$ and

$$
E_{1, k}\left(\left[a_{1}, \ldots, a_{k}\right] ; y_{1}, \ldots, y_{k}\right)=\left[a_{1} \diamond y_{1}, \ldots, a_{k} \diamond y_{k}\right],
$$

here we use the notation $a \diamond\left(b_{1}, \ldots, b_{s}\right)=\left(a^{(1)} \cdot b_{1}, \ldots, a^{(s)} \cdot b_{s}\right)$, so using this notation $[a] \smile_{1}\left[b_{1}, \ldots, b_{s}\right]=\left[a \diamond\left(b_{1}, \ldots, b_{s}\right)\right]$. Further iteration by (14) gives the general formula

$$
\begin{gathered}
E_{1, k}\left(\left[a_{1}, \ldots, a_{n}\right] ; y_{1}, \ldots, y_{k}\right)= \\
\sum\left[a_{1}, \ldots, a_{i_{1}-1}, a_{i_{1}} \diamond y_{1}, a_{i_{1}+1}, \ldots, a_{i_{k}-1}, a_{i_{k}} \diamond y_{k}, a_{i_{k}+1}, \ldots, a_{n}\right],
\end{gathered}
$$

where the summation is taken over all $1 \leqslant i_{1}<\ldots<i_{k} \leqslant n$.

Of course, so defined, the operations $E_{1, k}$ automatically satisfy the condition (14). It remains to prove the

Proposition 3. The operations $E_{1, k}$ satisfy the conditions (13) and (15).

Proof. The condition (13) is trivial for $x=[a]$ of length 1 and $k>2$. For $x=[a]$ and $k=2$ this condition degenerates to

$$
E_{1,1}\left([a] ; y_{1} \cdot y_{2}\right)+y_{1} \cdot E_{1,1}\left([a] ; y_{2}\right)-E_{1,1}\left([a] ; y_{1}\right) \cdot y_{2}=0
$$

and this equality easily follows from the definition of $E_{1,1}=\smile_{1}$. For a long $x=$ $\left[a_{1}, \ldots, a_{m}\right]$ the condition (13) can be checked by induction on the length $m$ of $x$ using the condition (14).

Similarly, the condition (15) is trivial for $x=[a]$ of length 1 unless the case $m=n=1$ and in this case this condition degenerates to

$$
\left.E_{1,1}\left(E_{1,1}(x ; y) ; z\right)=E_{1,1}\left(x ; E_{1,1}(y) ; z\right)\right)+E_{1,2}(x ; y, z)+E_{1,2}(x ; z, y) .
$$

This equality easily follows from the definition of $E_{1,1}=\smile_{1}$. For a long $x=$ $\left[a_{1}, \ldots, a_{m}\right]$ the condition (15) can be checked by induction on the length $m$ of $x$ using the condition (14).

Remark 1. For a DG-coalgebra $(A, d, \Delta: A \rightarrow A \otimes A)$ there is a standard $D G$ coalgebra map $g_{A}: A \rightarrow B \Omega A$ from $A$ to the bar of cobar of $A$. This map is the coextension of the universal twisting cochain $\phi_{A}: A \rightarrow \Omega A$ defined by $\phi(a)=[a]$ and is a weak equivalence, i.e. it induces an isomorphism of homology. Suppose $A$ is a DG-bialgebra. Then the constructed sequence of operations $E_{1, k}$ define a multiplication $\mu_{E}: B \Omega A \otimes B \Omega A \rightarrow B \Omega A$ on the bar construction $B \Omega A$ so that it becomes a DG-bialgebra. Direct inspection shows that $g_{A}: A \rightarrow B \Omega A$ is multiplicative, so it is a weak equivalence of DG-bialgebras. Dualizing this statement we obtain a weak equivalence of DG-bialgebras $\Omega B A \rightarrow A$ which can be considered as a free (as an algebra) resolution of a $D G$-bialgebra $A$. 


\section{References}

[1] J. Adams, On the non-existence of elements of Hopf invariant one, Ann. Math. 72 (1960), 20-104.

[2] H. Baues, The double bar and cobar construction, Compositio Math.43 (1981), 331-341.

[3] C. Berger, B. Fresse, Combinatorial operad action on cochains, preprint (2001), math.AT/0109158.

[4] N. Berikashvili, On the differentials of spectral sequence, Proc. Tbil. Math. Inst., 51 (1976), 1-105.

[5] E. Brown, Twisted tensor products. Ann. of Math., 69 (1959), 223-246.

[6] M. Gerstenhaber, The cohomology structure of an associative ring, Ann. of Math., 78 (1963), 267-288.

[7] M. Gerstenhaber, On the deformations of rings and algebras, Ann. of Math., 79, 1 (1964), 59-103.

[8] M. Gerstenhaber and A. Voronov, Higher operations on Hochschild complex, Functional Annal. Appl. 29 (1995), 1-6.

[9] E. Getzler, Cartan homotopy formulas and the Gauss-Manin connection in cyclic homology, Israel Math. Conf. proc. 7 (1993), 65-78.

[10] E. Getzler and J.D. Jones, Operads, homotopy algebra, and iterated integrals for double loop spaces, preprint (1994), hep-th/9403055.

[11] T. Kadeishvili, The $A(\infty)$-algebra Structure and Cohomology of Hochschild and Harrison, Proc. of Tbil. Math. Inst., 91, (1988), 19-27.

[12] T. Kadeishvili and S. Saneblidze, On a multiplicative model of a fibration, Bull. of Georg. Acad. Sci., 153 (1996), 345-346.

[13] T. Kadeishvili and S. Saneblidze, The twisted Cartesian model for the double path space fibration, preprint (2002), math. AT/021022.

[14] L. Khelaia, On some chain operations, Bull. Georg. Acad. Sci. 96, 3 (1979), 529-531.

[15] J. McLure, J. Smith, A solution of Deligne's conjecture, preprint (1999), math.QA/9910126.

[16] J. McLure, J. Smith, Multivariable cochain operations and little n-cubes, preprint (2001), math.QA/0106024.

[17] H.J. Munkholm, The Eilenberg-Moore spectral sequence and strongly homotopy multiplicative maps, J.P.A.A., 5 (1974), 1-50.

[18] S. Saneblidze, The Hohchschild complex of a space is the complex of the Hochschild set, preprint (2000). 
[19] J.D. Stasheff, Homotopy associativity of H-spaces. I, II, Trans. Amer. Math. Soc., 108 (1963), 275-312.

[20] B. Ndombol and J.-C. Thomas, Products in the Hochschild homology of spaces, Preprint (1998).

[21] A. Voronov, Homotopy Gerstenhaber algebras, preprint (1999), math.QA/9908040.

This article may be accessed via WWW at http://www.rmi.acnet.ge/hha/ or by anonymous ftp at

ftp://ftp.rmi.acnet.ge/pub/hha/volumes/2005/n2a6/v7n2a6.(dvi,ps,pdf)

T. Kadeishvili kade@rmi.acnet.ge

A. Razmadze Mathematical Institute

Georgian Academy of Sciences

M. Aleksidze st., 1

0193 Tbilisi,

Georgia 